\title{
Energetics of runaway electrons during tokamak disruptions
}

\author{
J. Riemann ${ }^{a}$, H. M. Smith ${ }^{a, b}$ and P. Helander ${ }^{a}$ \\ ${ }^{a}$ Max-Planck-Institut für Plasmaphysik, 17491 Greifswald, Germany \\ ${ }^{b}$ Max-Planck-Institut für Sonnensystemforschung, 37191 Katlenburg-Lindau, Germany
}

In a tokamak disruption, a substantial fraction of the plasma current can be converted into runaway electrons. Although these are usually highly relativistic, their total energy is initially much smaller than that of the pre-disruption plasma. However, following a suggestion by Putvinski et al. [Plasma Phys. Control. Fusion 39, B157 (1997)], it is shown that as the post-disruption plasma drifts toward the first wall, a non-negligible part of the energy contained in the poloidal magnetic field can be converted into kinetic energy of the runaway electrons. This process is simulated numerically, and it is found that in an ITER-like tokamak runaway electrons can gain kinetic energies up to about $70 \mathrm{MJ}$ by this mechanism.

PACS: 52.55.Fa, 52.35.Py, 52.25.Fi, 52.25.Tx, 52.20.Fs 


\section{INTRODUCTION}

When a tokamak plasma experiences a disruption, an inductive toroidal electric field arises which can accelerate "runaway" electrons to relativistic energies [1]. After the thermal quench, when the plasma has cooled down to a temperature of 5-10 eV, all the remaining current is carried by runaway electrons. In particular when the plasma current is large, a major fraction of it can be converted into runaway electrons in this way [2], and in ITER the expected current-conversion fraction is about $2 / 3[3,4]$. When the plasma current has thus been converted into a current of runaway electrons embedded in the cool post-disruption plasma, most of the plasma kinetic energy resides in the runaways, but this energy is much smaller than the pre-disruption thermal energy. It is also much smaller than the energy of the poloidal magnetic field, $B_{\theta}$, which per unit length of a cylindrical plasma column is

$$
W_{\mathrm{m}} \sim \int \frac{B_{\theta}^{2}}{2 \mu_{0}} 2 \pi r d r \sim \frac{\mu_{0} I^{2}}{4 \pi}
$$

If the current $I$ is carried by relativistic electrons of energy $\gamma m_{\mathrm{e}} c^{2}$, the corresponding kinetic energy is

$$
W_{\mathrm{k}} \sim \frac{I(\gamma-1) m_{\mathrm{e}} c}{e}
$$

so that

$$
\frac{W_{\mathrm{m}}}{W_{\mathrm{k}}} \sim \frac{I}{(\gamma-1) I_{\mathrm{A}}}
$$

where $I_{\mathrm{A}}=4 \pi m_{\mathrm{e}} c / \mu_{0} e=17 \mathrm{kA}$ is the Alfvén current. In large tokamaks, such as ITER, most runaway electrons are produced in avalanches caused by collisions at close range between existing runaways and thermal electrons [5-7], in which case the average Lorentz factor becomes $\gamma \sim 2 \ln \Lambda$ [2]. After a disruption in ITER we thus expect most of the "free" energy to reside in the poloidal magnetic field, $W_{\mathrm{m}} / W_{\mathrm{k}} \gg 1$. Putvinski et al. [8] have suggested that some of this energy could be transferred to the runaway electrons when the plasma drifts toward the wall. This suggestion was substantiated by a simple one-dimensional model calculation, where a cylindrical plasma was surrounded by a cylindrical wall with a radius that decreased linearly with time. The current profile was evolved in time, and it was found that a large fraction of the magnetic energy could indeed be converted into kinetic energy of runaway electrons, particularly if the shrinkage of the plasma region was slow. In a very recent article [9], Loarte et al. reported experimental evidence that such conversion indeed takes place in the Joint European Torus (JET), and this conclusion was again supplemented 
by one-dimensional modelling. In the present paper, we take the next theoretical step and perform two-dimensional (axisymmetric) modelling, still taking the plasma to have a circular cross section but calculating its vertical movement self-consistently with the resistive diffusion in conducting structures exterior to the plasma. The paper is organized as follows. In Sec. II, a simple argument is presented as to why a slow movement of the plasma results in a large fraction of the energy being deposited there (rather than in the exterior conductors). The mathematical model that underlies our numerical calculations is described in Secs. III-IV, and in Sec. V our numerical results are presented.

\section{MODEL PROBLEM}

In a typical disruption, the thermal quench lasts for less than $1 \mathrm{~ms}$ (perhaps slightly longer in ITER), and is followed either by a quick current quench or by a partial conversion of the Ohmic current to runaway electrons. The latter do not appear in all tokamaks or in all disruptions, but it is believed that they are more likely to arise in devices with large plasma current, because in such plasmas there is a very efficient runaway generation mechanism caused by close collisions between fast and slow electrons. This so-called avalanche process is discussed in detail in Refs. [1-8]. In the next stage of the disruption the runaway plasma usually drifts toward the wall, where it induces eddy currents, particularly when the plasma starts touching the wall and the current is being scraped off. This also causes a backreaction on the plasma in the form of an induced toroidal electric field, which deposits energy in the plasma current and thus accelerates the runaway electrons further. The energy of the poloidal magnetic field is therefore dissipated partly in the plasma and partly in the conducting structures that surround it. If the plasma moves slowly toward the wall, most of the energy is deposited in the plasma itself, and if it moves quickly the energy instead ends up in the external conductors. To understand why, it is helpful to consider a simple model where the plasma and the wall are represented by two coupled electric circuits. If their inductances are $L_{1}$ and $L_{2}$, respectively, the currents $I_{1}$ and $I_{2}$, the mutual inductance $M$, and the resistance of the wall is $R_{2}$, the voltage becomes

$$
U_{1}=-L_{1} \dot{I}_{1}-M \dot{I}_{2}
$$


in the plasma and

$$
U_{2}=R_{2} I_{2}=-L_{2} \dot{I}_{2}-M \dot{I}_{1}
$$

in the wall. If the plasma current decays (in practice because it is scraped off as the plasma moves into the wall), e.g., like

$$
I_{1}(t)=I_{0} e^{-t / t_{0}},
$$

and there is no initial wall current, $I_{2}(0)=0$, then

$$
I_{2}(t)=\frac{M I_{0}}{L_{2}\left(\omega t_{0}-1\right)}\left(e^{-t / t_{0}}-e^{-\omega t}\right)
$$

where $\omega=R_{2} / L_{2}$ is the natural decay rate of the wall current. The total energy deposited in the plasma is thus

$$
W_{1}=\int_{0}^{\infty} U_{1} I_{1} d t=\left(L_{1}-\frac{M^{2}}{L_{2}\left(\omega t_{0}+1\right)}\right) \frac{I_{0}^{2}}{2},
$$

and that in the wall becomes

$$
W_{2}=\int_{0}^{\infty} R_{2} I_{2}^{2} d t=\frac{M^{2} I_{0}^{2}}{2 L_{2}\left(\omega t_{0}+1\right)},
$$

so that

$$
W_{1}+W_{2}=\frac{L_{1} I_{0}^{2}}{2}
$$

as expected. If the plasma current decays slowly, $\omega t_{0} \gg 1$, then nearly all the energy is dissipated in the plasma since the current induced in the wall remains small at all times and the dissipation rate is proportional to the square of this current. In the opposite limit of a very rapidly decaying plasma current, $\omega t_{0} \ll 1$, a fraction

$$
\frac{W_{1}}{W_{1}+W_{2}}=1-\frac{M^{2}}{L_{1} L_{2}}
$$

of the energy ends up in the plasma.

\section{TWO-DIMENSIONAL MATHEMATICAL MODEL}

We now consider a much more accurate description of the system, which will form the basis of the numerical simulations described in the next section. Our aim is to solve for the evolution of the magnetic field in the plasma, the vacuum region and the surrounding 
conducting structures. The geometry is taken to be axisymmetric, so that the magnetic field can be written as

$$
\mathbf{B}=I(\psi, t) \nabla \varphi+\nabla \varphi \times \nabla \psi
$$

where $\psi(R, z, t)$ is proportional to the poloidal magnetic flux and $(R, \varphi, z)$ are cylindrical coordinates. The toroidal current density is

$$
J_{\varphi}=\frac{\Delta^{*} \psi}{\mu_{0} R}
$$

with

$$
\Delta^{*}=R \frac{\partial}{\partial R} \frac{1}{R} \frac{\partial}{\partial R}+\frac{\partial^{2}}{\partial z^{2}}
$$

and is taken to be the sum of the Ohmic current $\sigma E_{\varphi}$ and the runaway current

$$
J_{\varphi}=\frac{\sigma}{R} \frac{\partial \psi}{\partial t}+J_{\mathrm{r}}
$$

so that the Grad-Shafranov equation becomes

$$
\sigma \mu_{0} \frac{\partial \psi}{\partial t}=\Delta^{*} \psi-\mu_{0} R J_{\mathrm{r}}
$$

This equation is to be solved as an initial-value problem subject to the boundary condition that $\psi \rightarrow 0$ at infinity. In practice, the computational domain is finite and we then require that the normal derivative of $\psi$ should vanish on the boundary. The runaway current obeys an unconventional Ohm's law since the runaway electrons already move almost at the speed of light and thus cannot be accelerated further by the electric field. Instead, they can create more runaways in collisions with thermal electrons, so that the time derivative of the runaway current (rather than the current itself) is proportional to the electric field. Accordingly, it can be shown that $[2]$

$$
\frac{\partial J_{\mathrm{r}}}{\partial t} \simeq \frac{J_{\mathrm{r}}}{\tau_{\mathrm{a}}}\left(\frac{\left\langle E_{\varphi}^{\prime}\right\rangle}{E_{\mathrm{c}}}-1\right)
$$

where $\left\langle E_{\varphi}^{\prime}\right\rangle$ is the flux-surface-averaged electric field in a frame moving with velocity $\mathbf{v}$,

$$
E_{\varphi}^{\prime}=\left(\frac{\partial}{\partial t}+\mathbf{v} \cdot \nabla\right) \frac{\psi}{R}
$$

Furthermore

$$
\tau_{\mathrm{a}}=\tau \ln \Lambda \sqrt{\frac{3\left(Z_{\mathrm{eff}}+5\right)}{\pi \gamma(\epsilon)}}\left(1-\frac{E_{\mathrm{c}}}{E}+\frac{4 \pi\left(Z_{\mathrm{eff}}+1\right)^{2}}{3 \gamma(\epsilon)\left(Z_{\mathrm{eff}}+5\right)\left(E^{2} / E_{\mathrm{c}}^{2}+4 / \gamma^{2}(\epsilon)-1\right)}\right)^{1 / 2}
$$


with $\gamma(\epsilon)=(1+1.46 \sqrt{\epsilon}+1.72 \epsilon)^{-1}$ and $\epsilon=r / R$ is the runaway avalanche time [2],

$$
\tau=\frac{4 \pi \epsilon_{0}^{2} m_{\mathrm{e}} c^{3}}{n e^{4} \ln \Lambda}
$$

the collision time for relativistic electrons [10] and

$$
E_{\mathrm{c}}=\frac{m_{\mathrm{e}} c}{e \tau}
$$

the critical electric field below which no runaway acceleration occurs [11].

Equation (2) is only valid if the electric field exceeds $E_{\mathrm{c}}$; if $\left\langle E_{\varphi}^{\prime}\right\rangle<E_{\mathrm{c}}$ the runaway current is damped because of collisional friction and emission of synchrotron radiation. The evolution of the current density is modelled as

$$
\frac{1}{J_{\mathrm{r}}} \frac{\partial J_{\mathrm{r}}}{\partial t} \simeq \frac{1}{\tau_{\mathrm{a}}}\left(\frac{\left\langle E_{\varphi}^{\prime}\right\rangle}{E_{\mathrm{c}}}-1\right) \Theta\left(\left\langle E_{\varphi}^{\prime}\right\rangle-E_{\mathrm{c}}\right)+\frac{1}{\tau_{\mathrm{d}}}\left(\frac{\left\langle E_{\varphi}^{\prime}\right\rangle}{E_{\mathrm{c}}}-1\right) \Theta\left(E_{\mathrm{c}}-\left\langle E_{\varphi}^{\prime}\right\rangle\right),
$$

where $\tau_{d}=2 \tau \ln \Lambda$ represents the typical damping time caused by collisional friction. On longer time scales than those encountered in the present context, pitch-angle scattering and synchrotron radiation can be important [12]. For flux surfaces that intersect the wall, Eq. (4) is replaced by $J_{\mathrm{r}}=0$ in the calculation.

In principle, the plasma should occupy the region $V$ inside a given flux surface, i.e., a level surface of $\psi$ that varies with time. We shall simplify the problem by approximating this region by a large-aspect-ratio-torus with circular cross section. This eases the computational burden significantly, because we thus avoid having to calculate how the shape of the plasma evolves and instead only need to determine its overall position. The minor radius of the plasma remains constant $(=a)$ until it touches the wall and starts to be scraped off. To complete the model, we then only need to specify the velocity $\mathbf{v}$, which we will take to be vertical in order to simulate a vertical disruption. Its magnitude is determined by the condition that the net magnetic force on the plasma,

$$
\mathbf{F}=\int_{V} \mathbf{J} \times \mathbf{B} d V
$$

should vanish, since the plasma inertia is very small. In order to calculate this force, it is convenient to split the current into two terms,

$$
\mathbf{J}=\mathbf{J}_{0}+\mathbf{J}_{1}
$$


where $\mathbf{J}_{0}$ denotes the current in the exterior conductors and $\mathbf{J}_{1}$ the plasma current. The magnetic field can be decomposed correspondingly

$$
\mathbf{B}=\mathbf{B}_{0}+\mathbf{B}_{1}
$$

where $\nabla \times \mathbf{B}_{i}=\mu_{0} \mathbf{J}_{i}$. The force then becomes

$$
\mathbf{F}=\int_{V} \mathbf{J}_{1} \times\left(\mathbf{B}_{0}+\mathbf{B}_{1}\right) d V
$$

where the self-force

$$
\int \mathbf{J}_{1} \times \mathbf{B}_{1} d V=\mu_{0}^{-1} \int \nabla \cdot\left(\mathbf{B}_{1} \mathbf{B}_{1}-\mathbf{I} B_{1}^{2} / 2\right) d V
$$

vanishes since the domain of integration can be extended to infinity without affecting the result. In order to simplify the remaining force, we take the aspect ratio $\epsilon^{-1}$ of the plasma torus to be large. In the vertical force density

$$
\left(\mathbf{J}_{1} \times \mathbf{B}_{0}\right) \cdot \nabla z=J_{1 R} B_{0 \varphi}-J_{1 \varphi} B_{0 R}
$$

the first term on the right is then small, because writing $B_{0 \varphi}=I_{0} / R$ gives

$$
\int_{V} R J_{1 R} B_{0 \varphi} d V=I_{0} \int_{V} \mathbf{J}_{1} \cdot \nabla R d V=I_{0} \int_{\partial V} R \mathbf{J}_{1} \cdot d \mathbf{S}=0
$$

where $\partial V$ denotes the boundary of $V$, so that

$$
\int_{V} R J_{1 R} B_{0 \varphi} d V \sim \epsilon J_{1 R} B_{0 \varphi} V
$$

and thus

$$
\frac{\int R J_{1 R} B_{0 \varphi} d V}{\int R J_{1 \varphi} B_{0 R} d V} \sim \epsilon \ll 1,
$$

since we expect $J_{1 R} / J_{1 \varphi} \sim \epsilon$. In leading order, the vertical force is thus determined by the toroidal component of the plasma current

$$
F_{z} \simeq-\int_{V} J_{1 \varphi} B_{0 R} d V
$$




\section{COMPUTATIONAL MODEL}

We now describe how the mathematical model just described has been implemented computationally. The goal is to obtain an estimate for the runaway electron energy that may strike the first wall during a disruption in an ITER-like tokamak. Since the disruption parameters can only be predicted with limited confidence, it is acceptable to make approximations. In addition to the assumptions of large aspect ratio and circular cross section already mentioned, we use a simplified representation of the poloidal field (PF) coils, assuming a simple set of pair-wise symmetric PF coils and currents, thus neglecting all finer details of plasma shaping. Furthermore we only apply an active current to the PFC1 coil set (see Figure 1). These currents are symmetric and co-oriented with the plasma current and would thereby cause an elongation of the plasma cross section which is neglected. All other PF coils initially have zero current and later only carry relatively small induced currents. This arrangement of PF coil currents will provide an unstable equilibrium if the plasma is placed exactly between the upper (PFC1U) and lower (PFC1L) coil. In a real setup it would be supplemented by counter-oriented currents in the remaining PF coils and additional currents in the central solenoid for positioning and shaping. We neglect the stabilizing effect from additional counter-oriented PF currents and thus consider our estimates as a worst-case model. As a further simplification, we only model the vertical movement of the plasma, ignoring any horizontal displacement so as to avoid having to solve a two-dimensional equation of motion. None of these approximations should affect the energy channelling into runaway electrons qualitatively. The central part of the numerical algorithm is a time-dependent solution of the parabolic equation (1), which can be cast into the finite-volume (FV) representation

$$
\int_{V} \nabla \cdot \boldsymbol{\Gamma}_{\psi} d V=\int_{V}\left(S_{\psi}-\sigma \mu_{0} \frac{\partial \psi}{\partial t}\right) d V
$$

where $\boldsymbol{\Gamma}_{\psi}$ is the total flux of quantity $\psi$ and $S_{\psi}$ is a general source independent of time. In this form, the equation can be solved with the FV transport code BoRiS using Newton's method [13].

The numerical simulation is performed on a rectangular subdomain $\left(\varepsilon \leq R \leq 3 R_{0}\right.$, $-3 R_{0} \leq z \leq 3 R_{0}$ ) of the $R-z$ plane containing all the major conducting structures where significant toroidal currents may flow, including the plasma region, vessel walls, PF coils and the central solenoid. These are chosen to resemble those in ITER and are shown in Fig. 1. 
The boundary conditions imposed are $\psi=0$ along $R=\varepsilon$ and $\nabla \psi \cdot d \mathbf{S}=0$ elsewhere.

All the model elements are stationary with the exception of the plasma, but instead of moving the plasma it proved advantageous to keep the plasma center fixed at $\left(R_{0}, 0\right)$ at all times and instead move all other objects with the velocity $v_{z}=-v_{\text {plas }}$. This practice enables to use a $2 \mathrm{D}$ non-equidistant grid that is finest at the plasma center to achieve an almost constant angular resolution for individual flux surfaces contributing to averages of the induced electric field strength $\left\langle E_{\varphi}^{\prime}\right\rangle$.

The main quantity of interest is the fraction of magnetic field energy that is converted into kinetic runaway electron energy during the vertical motion of the plasma toward the vessel wall, where this energy is deposited as the current is scraped off. In the initial state of each simulation the coil currents are taken to be up-down symmetric and the plasma is positioned at the vertical center of the computational domain. The completely symmetric case, with the plasma in the middle, $z_{\text {plas }}=0$, is usually an (unstable) equilibrium state. A vertical disruption is induced in the beginning of the simulation by a small instantaneous vertical displacement $\delta z$ of the plasma from the center. This causes eddy currents in the conducting structures, which in turn affect the motion of the plasma.

The vertical velocity will in general vary during the motion of the plasma and therefore needs to be determined self-consistently from the vertical net force (5) on the plasma. Since plasma inertia is negligibly small the velocity can be determined from the condition $F_{z}=0$ by an iterative procedure that minimizes the vertical force as a result of a virtual time step performed with a guessed velocity. The virtual time step and the velocity guess are accepted if a given convergence criterion $\left(\left|F_{z}\right|<1 N\right.$ or $\left.\left|\Delta v_{z} / v_{z}\right|<10^{-3}\right)$ is satisfied. Thus the timedependent solution of Eq. (1) becomes a sequence of time-step guesses (outer iterations), each of them being brought to the prescribed numerical accuracy within a series of (inner) Newton iterations.

The total energy that is transferred to the plasma volume $V_{P}$ over the time $t$ is

$$
W_{\text {plas }}=\int_{0}^{t} d t^{\prime} \int_{V_{\mathrm{P}}} J_{\varphi} E_{\varphi} d V=\int_{0}^{t} d t^{\prime} \int_{V_{\mathrm{P}}}\left(\sigma E_{\varphi}+J_{\mathrm{r}}\right) E_{\varphi} d V=W_{\Omega}+W_{\mathrm{r}}
$$

The term $W_{\Omega}$ involving the Spitzer conductivity $\sigma$ describes the energy deposited as plasma heating via the Ohmic current, and the second term $W_{\mathrm{r}}$ is the total amount of energy transferred to the runaways. The runaway electrons also suffer energy losses, primarily 
through friction against the cold bulk electrons, so that the energy

$$
W_{E_{\mathrm{c}}} \approx \int_{0}^{t} d t^{\prime} \int_{V_{\mathrm{P}}} J_{\mathrm{r}} E_{\mathrm{c}} d V
$$

is spent on maintaining the runaway current against collisional slowing-down. The final runaway energy that hits the vessel wall is thus given by

$$
W_{\mathrm{RE}}=W_{\mathrm{RE}}^{0}+\int_{0}^{t} d t^{\prime} \int_{V_{\mathrm{P}}} J_{\mathrm{r}}\left(E_{\varphi}-E_{\mathrm{c}}\right) d V=W_{\mathrm{RE}}^{0}+\Delta W_{\mathrm{RE}}
$$

The energy transferred onto the vessel wall or any conducting structure moving with the velocity $v_{z}$ relative to the plasma is obtained by integrating the Ohmic power over the corresponding subdomain, e.g.

$$
W_{\text {wall }}=\int_{0}^{t} d t^{\prime} \int_{\text {wall }} J_{\varphi} E_{\varphi}^{\prime} d V
$$

\section{RESULTS AND DISCUSSION}

The parameters of the modelling were chosen to reflect ITER in size, $R_{0} \sim 6 \mathrm{~m}, a \sim 2 \mathrm{~m}$, and expected post-disruption plasma parameters, $T \sim 5 \mathrm{eV}, n \sim 10^{21} \mathrm{~m}^{-3}$. The plasma current, $I_{\mathrm{P}} \sim 2 / 3 I_{\mathrm{P}}^{0} \sim 10 \mathrm{MA}$, was taken to be carried exclusively by runaway electrons in the beginning of each simulation. The initial kinetic energy $W_{\mathrm{RE}}^{0} \sim 20 \mathrm{MJ}$ of the runaway electrons is about $3 \%$ of the energy in the corresponding poloidal magnetic field.

Figure 2 shows trajectories $z(t)$ of the plasma center as it moves toward the upper vessel wall after being initially displaced by $\delta z=0.25 \mathrm{~m}$ from its (vertically unstable) equilibrium position. The different curves marked $\mathrm{A}$ and $\mathrm{B}$ in the figure correspond to different (but up-down symmetric) PF-coil currents in the PFC1U/PFC1L coils, resulting in different degrees of vertical instability. Apparently, the motion of the plasma is qualitatively different before and after the time instant, $t=t^{*}$, when it first comes into direct contact with the wall. In the first, "free motion" phase, $t<t^{*}$, the plasma is initially accelerated and soon reaches a constant velocity $\left(v_{z} \sim 5-10 \mathrm{~m} / \mathrm{s}\right)$ which is determined by the balance between several destabilizing effects and the stabilizing influence of eddy currents that are induced in response to the plasma motion. The main destabilizing effect on the plasma position is the attractive force from the co-oriented PF coil currents (PFC1), which is a result of the initial 
vertical displacement $\delta z$. The plasma motion is also affected by the value of the critical field strength $E_{\mathrm{c}}$ and the free decay rate of the plasma current. This "natural decay rate" is in contrast to the usual $L / R$ time independent of the plasma temperature, since the current is carried by runaway electrons, whose collision frequency only depends on the background plasma density.

In the second phase, $t>t^{*}$, plasma current is scraped off by the wall and the plasma accelerates (up to a velocity $v_{z} \sim 10-300 \mathrm{~m} / \mathrm{s}$ ) due to the enhanced current loss. The growth of the RE kinetic energy $\Delta W_{\mathrm{RE}}=W_{\mathrm{RE}}(t)-W_{\mathrm{RE}}^{0}$ in the scrape-off phase is shown in Figure 3, where it is seen to reach a final value of about $70 \mathrm{MJ}$ (corresponding to about $11 \%$ of the magnetic field energy of the initial post-disruption current). It is interesting to note that this energy depends strongly on the total initial current (see below), but does not seem to depend very much on the total duration of the vertical motion, i.e. on the strength of the instability drive from the PF coil current. Comparing Figures 2 and 3 it is clear that most of the growth of runaway kinetic energy occurs after the point where the plasma first hits the vessel wall (Fig. 4) and is thus directly related to the subsequent loss of plasma current. As argued in Sec. II and found by Putvinski et al. [8] the energy $W_{\text {plas }}$ deposited in the plasma (rather than in the surrounding conductors) is expected to grow with the duration of the scrape-off phase. Figure 5 compares the total energy (6) transferred to the plasma $W_{\text {plas }}$ and the work (7) done against the critical field strength $W_{E_{\mathrm{c}}}$ (i.e., the friction against thermal plasma electrons) for the two simulated cases. Since the same amount of current is scraped-off in roughly the same time $(0.04 \mathrm{~s})$ in cases A and B, the final runaway energy gain $\Delta W_{\mathrm{RE}}$ is almost identical in the two cases, see Table I. During the scrape-off phase, the energy transfer is almost independent of the prior history of the discharge.

The very sharp onset of the energy growth in the cases A and B is due to the fact that a flat profile $\left(J_{\mathrm{R}}^{0}=\right.$ const) was used for the initial current density. For a peaked profile (e.g. $J_{\mathrm{R}}^{0} \sim\left(1-\varrho^{2}\right)^{n}$ and identical total current) - which is likely to occur as reported in $[3,9,14]$ - no differences are seen in the free-motion phase, but the runaway energy exhibits a soft transition between the two phases (see case $A^{*}$ in Fig. 4) and the scrape-off phase lasts a factor $\sim 2$ longer. However, because the profile is peaked, the "effective" scrape-off time is comparable to that in the flat profile case, and consequently the runaway energy reaches roughly the same final value for both profiles.

To understand why the runaway electrons mainly gain kinetic energy in the scrape-off 
phase, it is instructive to consider the profile of the electric field that is induced during the motion of the plasma, since it determines the evolution of the RE current. Figure 6 shows a series of radial profiles $\left\langle E_{\varphi}\right\rangle$ measured at different times before and after first plasmawall contact at $t=t^{*}$. The flux-surface averaged field is found to remain nearly constant $\left\langle E_{\varphi}\right\rangle \approx E_{\mathrm{c}}$ over the entire period $t<t^{*}$, and the runaway current therefore changes very slowly with time during the free motion phase. Since $\left\langle E_{\varphi}(0)\right\rangle \approx E_{\mathrm{c}}$, all the power in this way goes into heating the plasma and very little into kinetic RE energy. In reality the heating is likely to be compensated by radiation losses and the temperature thus remains low. Upon contact with the wall the plasma begins to get scraped off from the outermost flux surfaces, to which the system responds in accordance with Lenz's law by inducing a much larger field that amplifies the remaining $\mathrm{RE}$ current so as to compensate for the lost current, at the further expense of poloidal magnetic field energy. This happens primarily in the outer layer of the remaining plasma, so that the toroidal electric field and the runaway current profiles develop a hollow structure. The skin current thus created has a density several times higher than the initial current, as shown in Figure 7. In the center of the plasma, the inductive electric field is always found to be close to the critical field, $\left\langle E_{\varphi}(0)\right\rangle \approx E_{\mathrm{c}}$. This observation can be shown to be a characteristic feature of the process under consideration. If the displacement current is neglected in Maxwell's equations, we must necessarily have

$$
\nabla^{2} E=\mu_{0} \frac{\partial J_{\varphi}}{\partial t}
$$

and if most of the current is carried by runaway electrons, $J_{\varphi}=J_{\mathrm{r}}$, Eq. (2) holds,

$$
\frac{\partial J_{\mathrm{r}}}{\partial t} \simeq \frac{J_{\mathrm{r}}}{\tau_{\mathrm{a}}}\left(\frac{E}{E_{\mathrm{c}}}-1\right)
$$

(where we have ignore the flux-surface averaging) so that

$$
a^{2} \nabla^{2} E=\frac{a^{2} \mu_{0} J_{\mathrm{r}}}{\tau_{\mathrm{a}} E_{\mathrm{c}}}\left(E-E_{\mathrm{c}}\right)
$$

The right-hand side can be estimated as

$$
\frac{a^{2} \mu_{0} J_{\mathrm{r}}}{\tau_{\mathrm{a}} E_{\mathrm{c}}}\left(E-E_{\mathrm{c}}\right) \sim \frac{\mu_{0} I_{\mathrm{r}}}{\pi \tau_{\mathrm{a}} E_{\mathrm{c}}}\left(E-E_{\mathrm{c}}\right) \approx \frac{I_{\mathrm{r}}}{0.2 \mathrm{MA}}\left(E-E_{\mathrm{c}}\right)
$$

where $\pi \tau_{\mathrm{a}} E_{\mathrm{c}} / \mu_{0}=I_{\mathrm{A}} \ln \Lambda \sqrt{3(Z+5) / \pi} / 4 \approx 0.2 \mathrm{MA}$ (for $\ln \Lambda \approx 10$ ) was used. Since $I_{\mathrm{r}} \gg$ $0.2 \mathrm{MA}$, it is clear that either $E \approx E_{\mathrm{c}}$ or $\left(a^{2} \nabla^{2} E\right) / E \gg 1$. As seen in Figures 6 and 7 , the first case corresponds to the situation in most of the plasma core, and the latter to the 
runaway skin current seen in the plasma edge. As noticed by Putvinski et al. [8], the skin depth is $\lambda \sim 0.1 a$ independently of how quickly the plasma moves toward the wall.

As a remark, we note that since thus $\left\langle E_{\varphi}\right\rangle \approx E_{\mathrm{c}}$ in most of the plasma, it is important to use the full expression (3) for the avalanche time rather than its asymptotic limit for $E \gg E_{\mathrm{c}}$ often used in the literature. Our simulations suggest that the RE kinetic energy content is otherwise underestimated by about $50 \%$.

Finally it is of interest how the motion of the plasma and the energy transfer into the runaway population depend on plasma parameters. Figure 8 compares four different results using one of them (case A) as a reference point. Doubling either the temperature or the density of the post-disruption plasma has little effect on the motion itself and only leads to small differences in the final kinetic energy gain. The strongest effect is observed when the conductivity of the vessel wall is doubled. As expected this leads to a slowing-down of the plasma motion toward the wall and an increase of the kinetic energy gained by the runaways. This increase is due to the doubling of the ratio between the resistive wall time $\tau_{\text {wall }}$ and the avalanche growth time $\tau_{a}$ that governs the exponential amplification of the runaway current. As indicated earlier, simulations have shown that the amount of kinetic energy gained by the runaway electrons clearly depends upon the initial RE current $I_{\mathrm{P}}^{0}$ (see Figure 9) and its strength at the beginning of the scrape-off phase.

Since the initial kinetic energy of the runaways is $W_{\mathrm{RE}}^{0} \sim 20 \mathrm{MJ}$, the energy gains (8) calculated with our model would lead to a final kinetic runaway energy of the order $100 \mathrm{MJ}$, i.e., an energy amplification factor $\sim 3.5-5$.

\section{CONCLUSION}

As originally suggested by Putvinski et al. [8] and recently confirmed experimentally by Loarte et al. [9], we find that a substantial conversion of magnetic energy into kinetic runaway energy is likely when a post-disuption ITER-size plasma drifts toward the first wall. Most of the energy conversion happens during the phase when the plasma touches the wall and its current is being "scraped off". The amount of magnetic energy channelled into the runaway electrons depends on how quickly the plasma moves toward the wall. Two effects compete: if the movement is slow, the currents in the wall and other conducting structures exterior to the plasma are small and most of the magnetic energy is dissipated in 
the plasma, where it accelerates runaway electrons. However, if the movement is slow, there is also plenty of time for these runaways to be slowed down by collisional friction against the thermal plasma electrons. Before the plasma touches the wall these two effects are of similar magnitude, but in the scrape-off phase the former dominates, and a runaway skin current develops at the plasma edge. The amount of runaway electron energy striking the wall is found to be of the order of 100 MJ. Because of the great uncertainties surrounding a disruption, it is difficult to predict this energy accurately, but in any case it could lead to substantial wall damage since the losses of runaway electrons tend to be highly localized. 


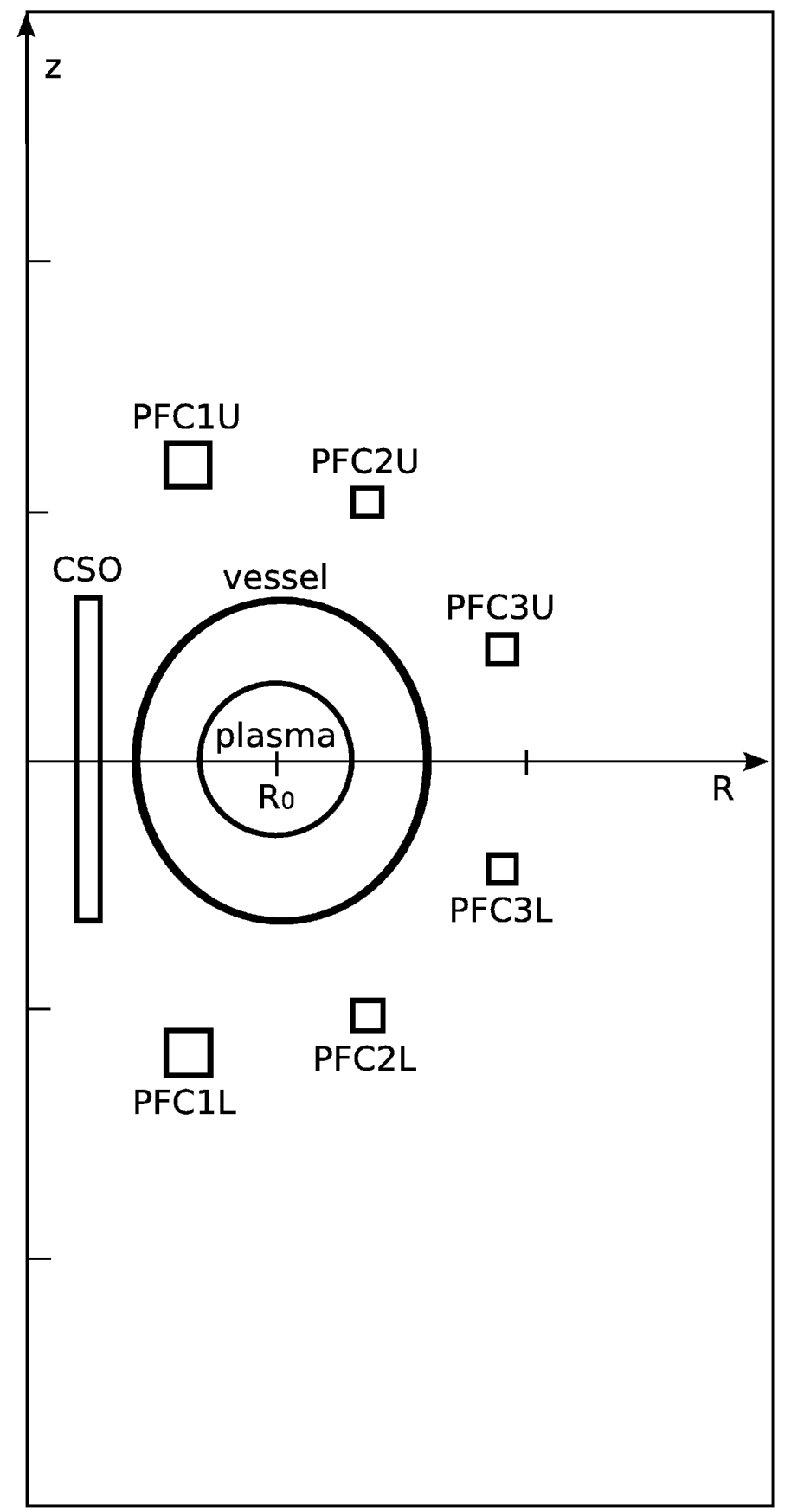

Figure 1: Computational domain with different objects (CSO central solenoid, PFC - poloidal field coil) as for the initial state. 


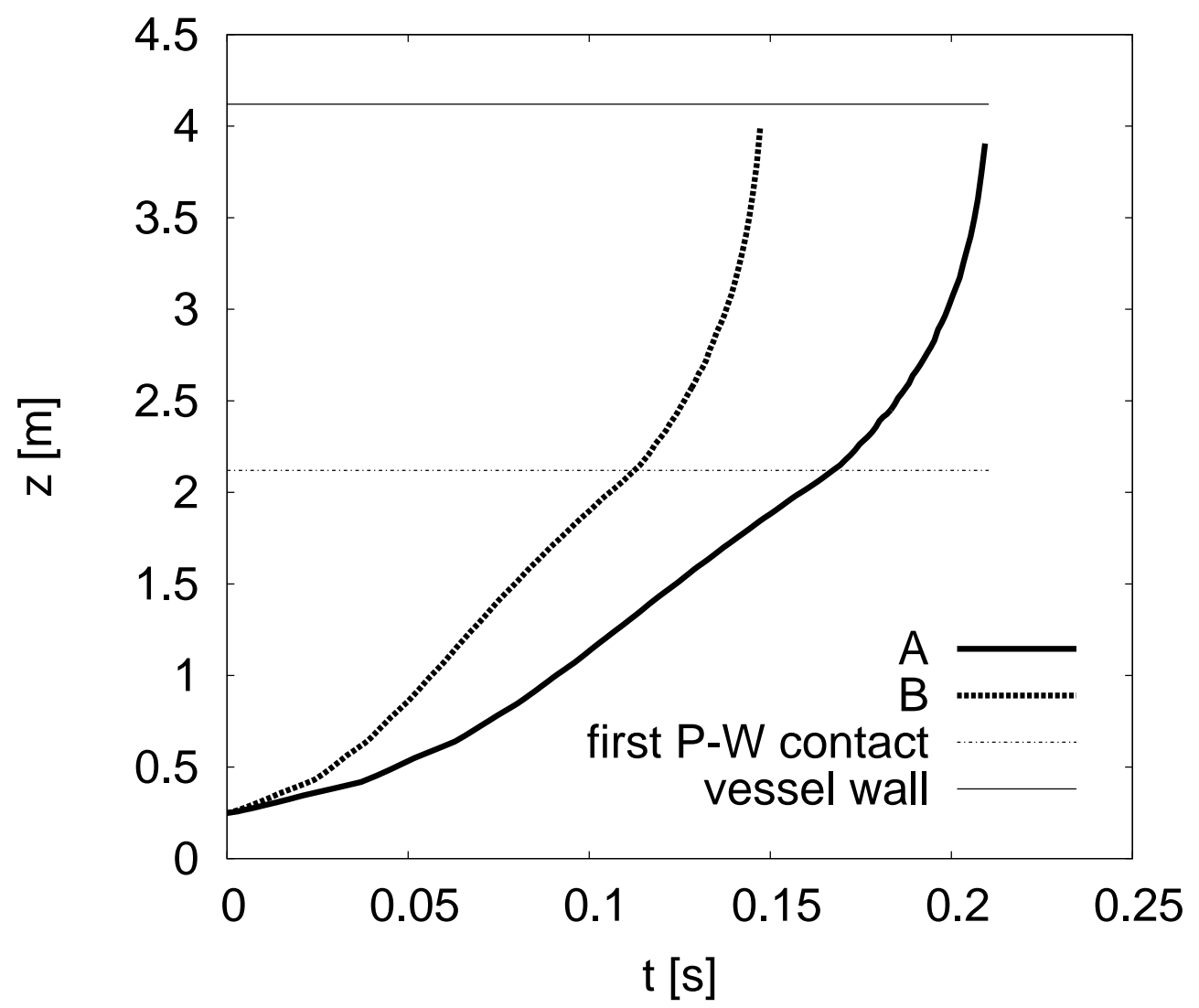

Figure 2: Vertical position $z$ of the plasma center as a function of time. The two curves correspond to different currents in the poloidal field coils PFC1U and PFC1L $\left(\mathrm{A}: I_{\mathrm{PFC} 1 \mathrm{U}}=I_{\mathrm{PFC} 1 \mathrm{~L}}=0.84 I_{\mathrm{P}}^{0}, \mathrm{~B}: I_{\mathrm{PFC} 1 \mathrm{U}}=I_{\mathrm{PFC} 1 \mathrm{~L}}=1.25 I_{\mathrm{P}}^{0}\right)$ assuming a pre-disruption plasma current $I_{\mathrm{P}}^{0}=15 \mathrm{MA}$. Plasma parameters are $T \sim 5 \mathrm{eV}$ and $n \sim 10^{21} \mathrm{~m}^{-3}$. For orientation the vertical position $(z=2.1)$ at which the plasma first hits the wall is indicated as well as the location of the upper vessel wall. After moving with almost constant velocity during the free-motion phase $(z<2.1)$, the plasma is strongly accelerated in the scrape-off phase $(z>2.1)$. 


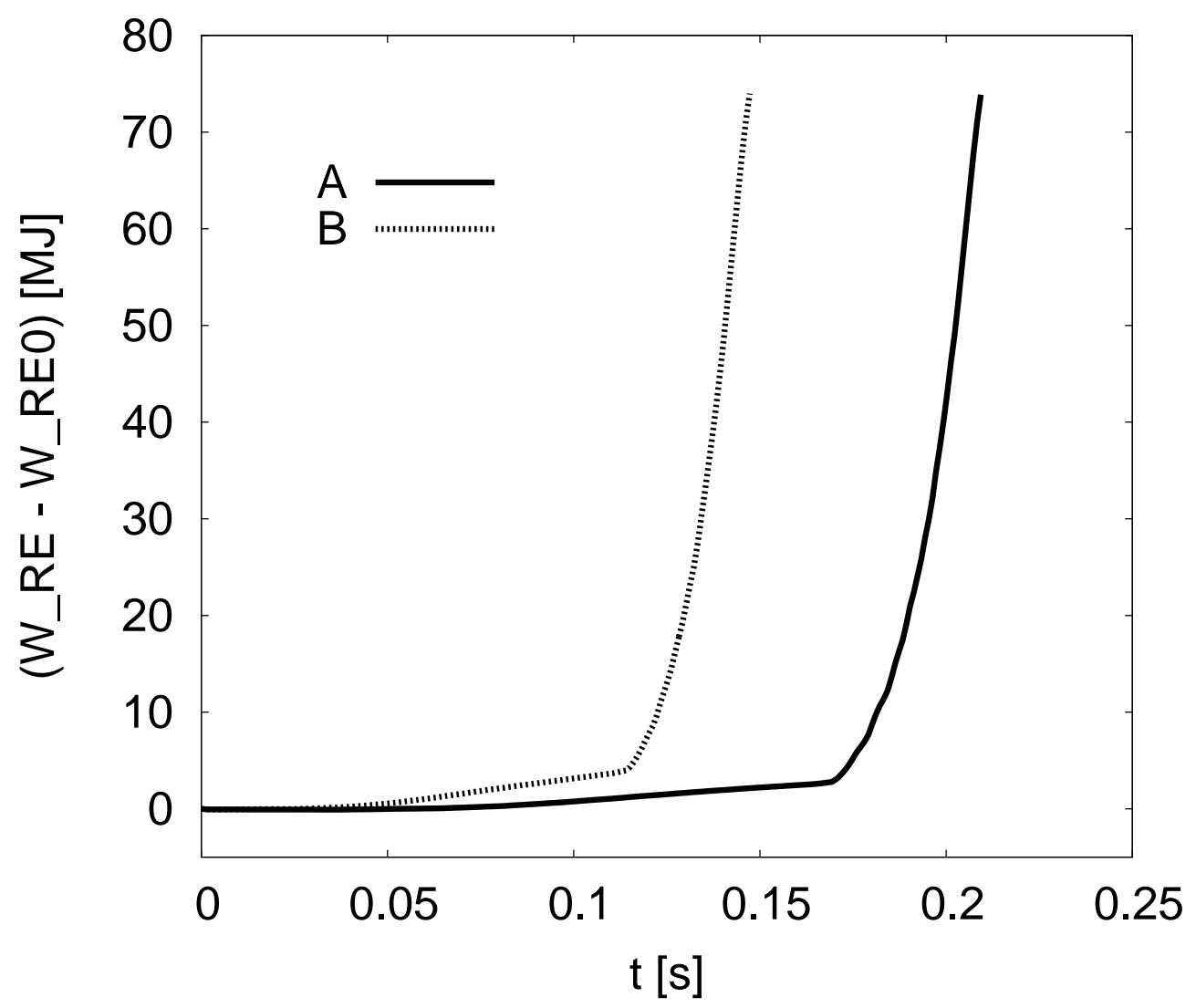

Figure 3: Growth of the RE kinetic energy $W_{\mathrm{RE}}-W_{\mathrm{RE}}^{0}$ as a function of time (for the two cases shown in Fig. 2). The RE kinetic energy is seen to grow significantly during the scrape-off phase and reaches approximately the same final level in the two cases. 


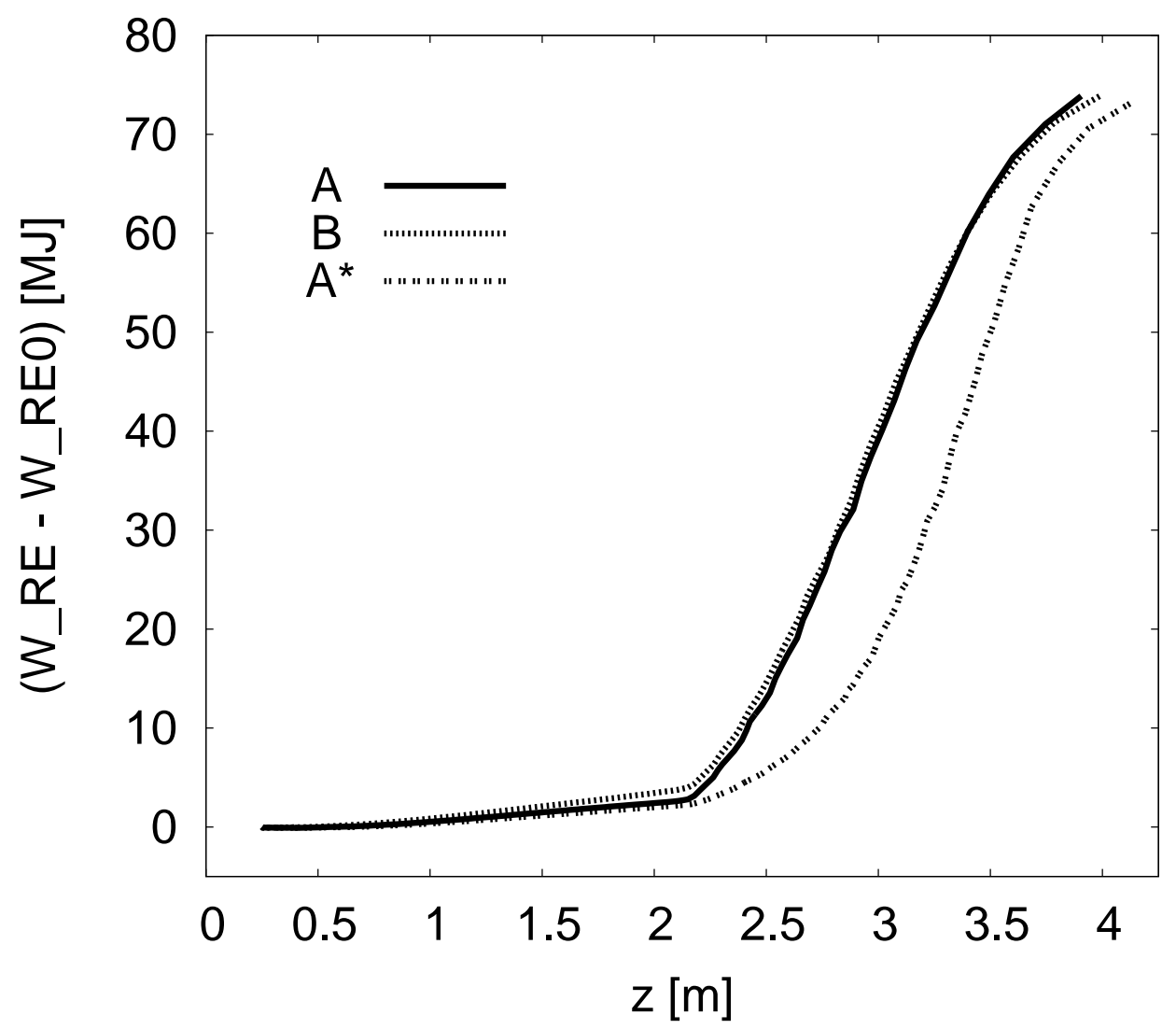

Figure 4: Growth of RE kinetic energy $W_{\mathrm{RE}}-W_{\mathrm{RE}}^{0}$ as a function of vertical plasma position in the two cases A and B depicted in Figs. 2 and 3, and with a more peaked current profile (case $\left.\mathrm{A}^{*}\right)$. 


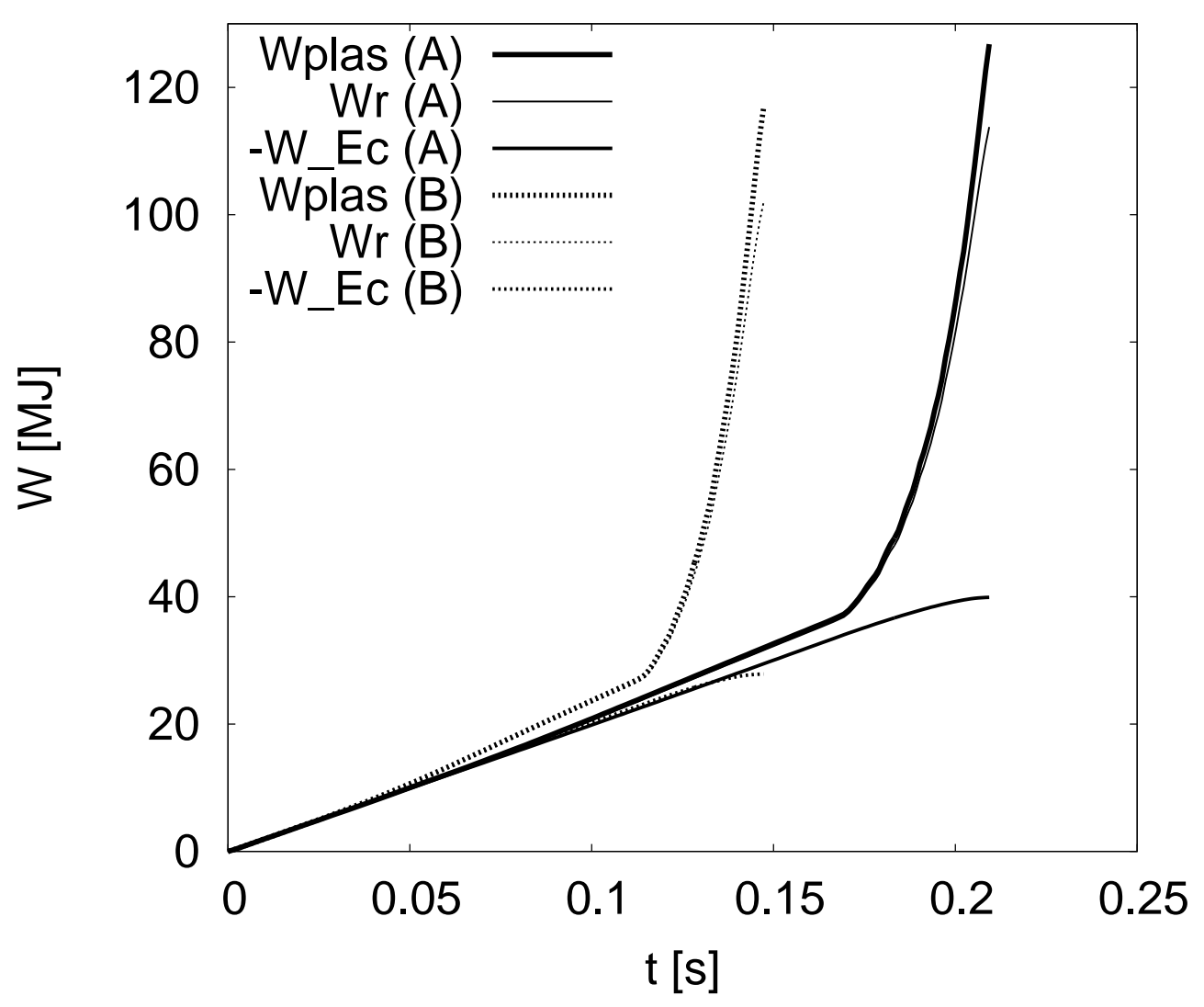

Figure 5: Different energies as a function of time (for the two cases described before). The total energy $W_{\mathrm{r}}$ channelled into the runaways is a monotonic function of time and is slightly smaller than the total energy $W_{\text {plas }}$ transferred to the plasma (6). Their difference is the energy $W_{\Omega}$ that goes into the Ohmic current carried by thermal plasma constituents. $W_{E_{c}}$ is the energy spent on work against the critical electric field strength $E_{c}$ in order to maintain the runaway current against collisional slowing-down. Although different amounts of energy are transferred to the plasma in the two cases, the final kinetic energy (8) gained by the runaways, $\Delta W_{\mathrm{RE}}=W_{\mathrm{r}}-W_{E_{c}}$ is almost identical. 


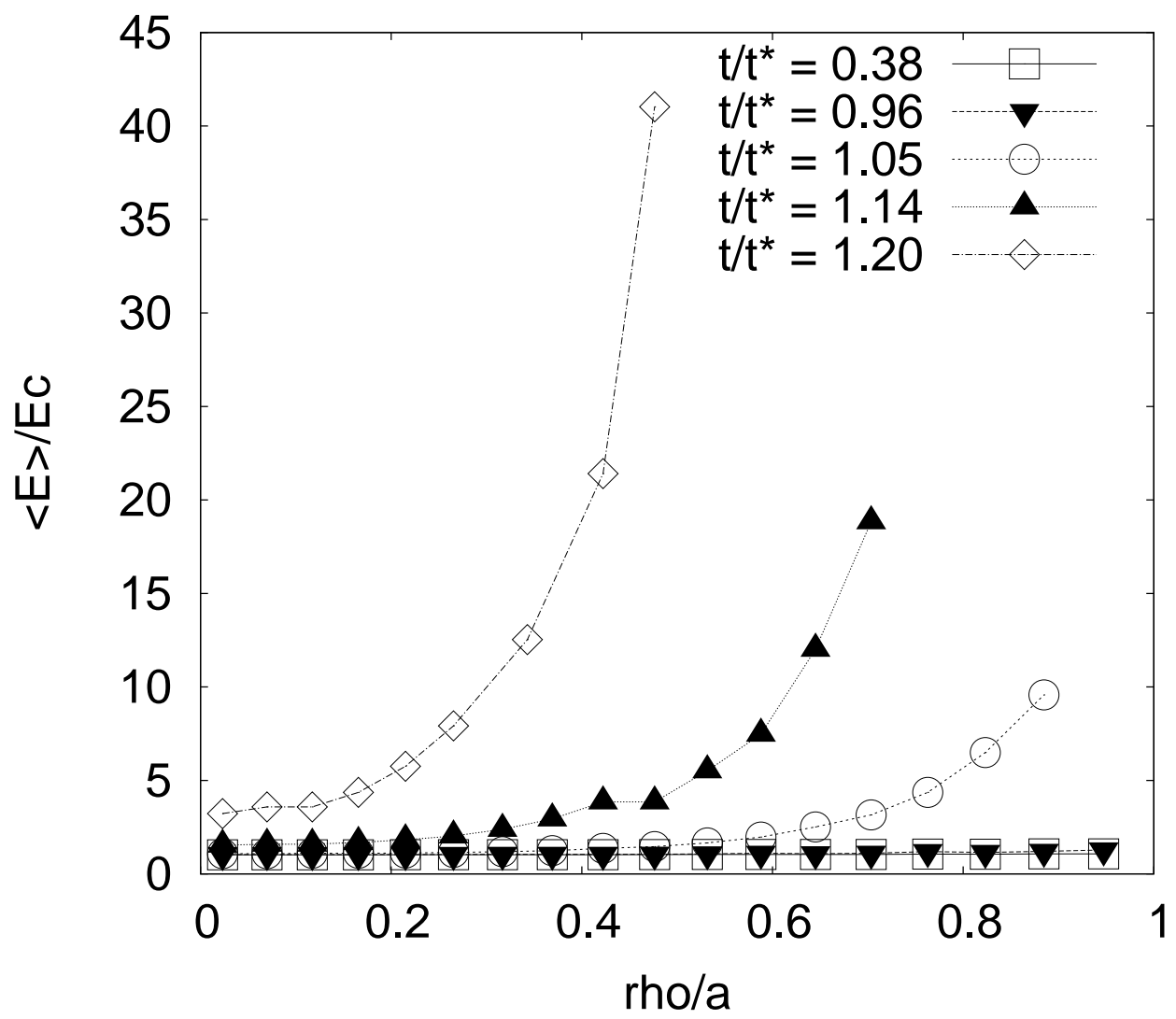

Figure 6: Flux-surface averaged electric field $\left\langle E_{\varphi}\right\rangle / E_{c}$ as a function of normalized plasma radius $\varrho / a$ at different (normalized) times $t / t^{*}$ for reference case A.

In the free-motion phase $t / t^{*}<1$ the electric field is almost constant at $\left\langle E_{\varphi}\right\rangle \approx E_{c}$. In the scrape-off phase the plasma cross section is seen to shrink while strong electric fields are induced at the edge and penetrate the plasma on the scale of the skin depth $\lambda \sim 0.1 a$. 


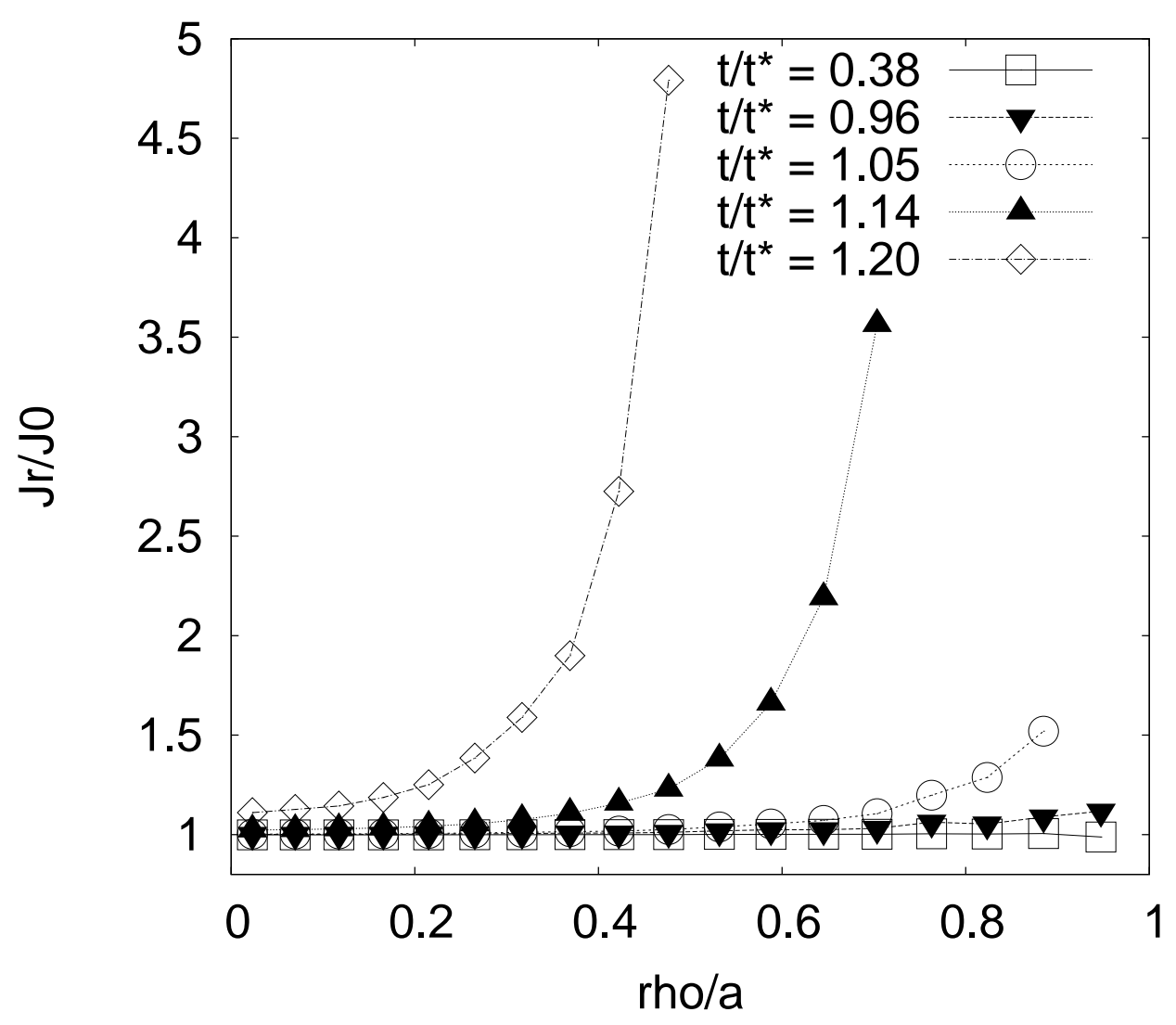

Figure 7: Normalized runaway current density $J_{r} / J_{0}$ as a function of normalized plasma radius $\varrho / a$ at different (normalized) times $t / t^{*}$ for reference case A.

The runaway current is seen to develop a hollow profile which reflects the effect of the electric fields that act as to compensate for the strong current losses in the scrape-off phase. 


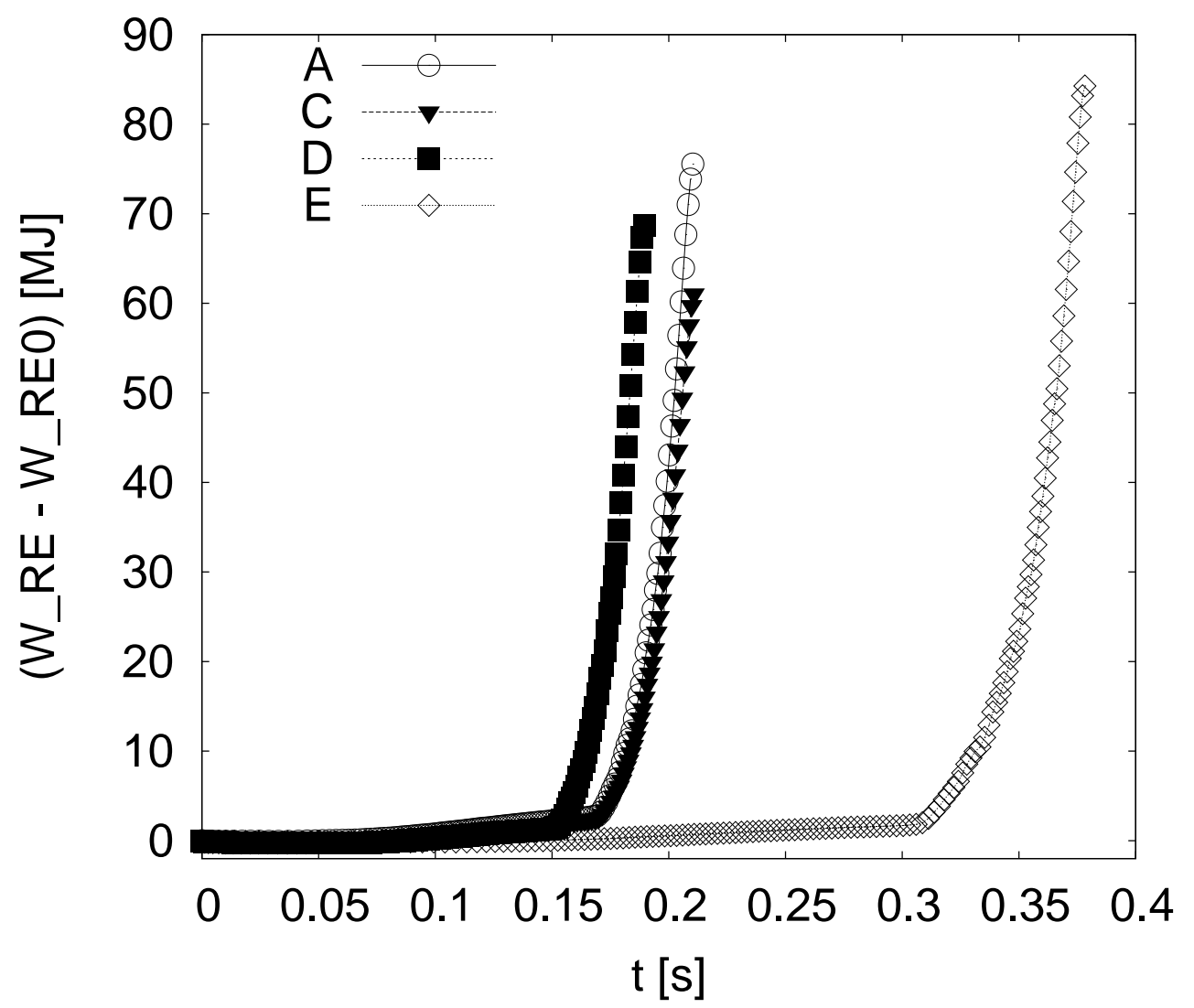

Figure 8: Dependence of the RE kinetic energy gain $\Delta W_{\mathrm{RE}}=W_{\mathrm{RE}}-W_{\mathrm{RE}}^{0}$ on different parameters. The figure compares four cases that are derived from reference case A by changing a single parameter at a time. Using $T \sim 10 \mathrm{eV}$ (instead of $5 \mathrm{eV}$ ) in case $\mathrm{C}$ reduces the penetration of electric fields into the plasma at the edge and thereby the kinetic energy gain to $\Delta W_{\mathrm{RE}}^{C} \approx 0.8 \Delta W_{\mathrm{RE}}^{A}$. For case $\mathrm{D}$, a higher density $n \sim 2 \cdot 10^{21} \mathrm{~m}^{-3}$ (instead of $n \sim 10^{21} \mathrm{~m}^{-3}$ ) leads to a higher critical field strength and thereby to a slight acceleration while almost keeping the kinetic energy gain at the same level as in case A. Case E illustrates the influence of doubling the conductivity of the vessel wall which results in a slower motion and a higher energy gain $\Delta W_{\mathrm{RE}}^{E} \approx 1.2 \Delta W_{\mathrm{RE}}^{A}$. This effect is due to the doubling of the resistive wall time $\tau_{\text {wall }}$. 


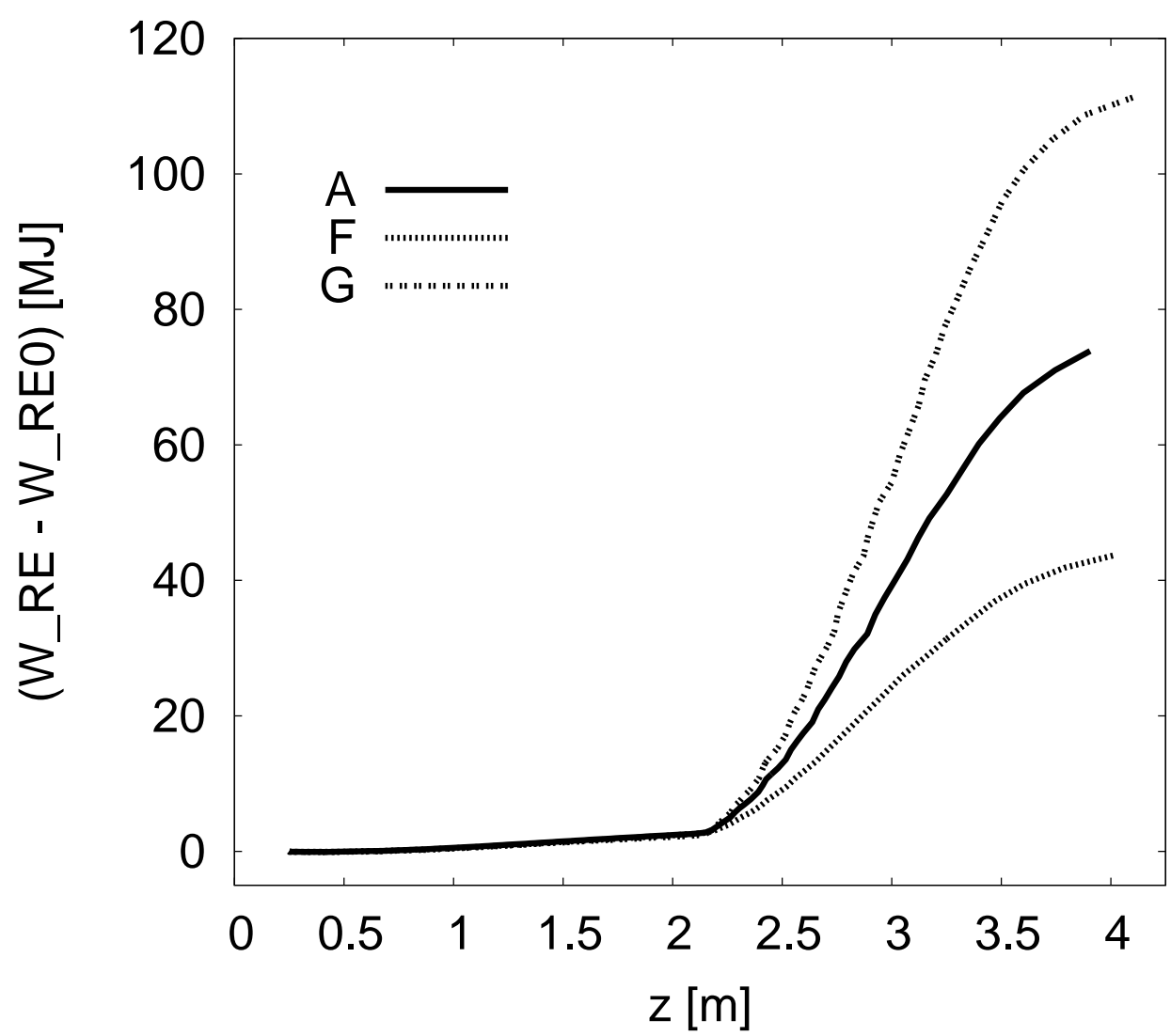

Figure 9: Dependence of the RE kinetic energy gain $\Delta W_{\mathrm{RE}}=W_{\mathrm{RE}}-W_{\mathrm{RE}}^{0}$ on the initial plasma current $I_{\mathrm{P}}^{0}$. The figure compares the reference case $\mathrm{A}\left(I_{\mathrm{P}}^{0}=10 \mathrm{MA}\right)$ with cases $\mathrm{F}\left(I_{\mathrm{P}}^{0}=7.5 \mathrm{MA}\right)$ and $\mathrm{G}\left(I_{\mathrm{P}}^{0}=12.5 \mathrm{MA}\right)$, assuming identical plasma cross sections carrying different RE current densities. 
TABLE I: scrape-off duration, total duration and RE energy gain for different cases

\begin{tabular}{|c|c|c|c|c|}
\hline case & $I_{\mathrm{P}}^{0}[\mathrm{MA}]$ & $\tau_{\text {scrape-off }}[\mathrm{s}]$ & $\tau_{\text {tot }}[\mathrm{s}]$ & $\Delta W_{\mathrm{RE}}[\mathrm{MJ}]$ \\
\hline $\mathrm{A}$ & 10 & 0.04 & 0.21 & 75 \\
\hline $\mathrm{A}^{*}$ & 10 & 0.09 & 0.24 & 75 \\
\hline $\mathrm{B}$ & 10 & 0.04 & 0.15 & 75 \\
\hline $\mathrm{C}$ & 10 & 0.04 & 0.21 & 61 \\
\hline $\mathrm{D}$ & 10 & 0.04 & 0.19 & 70 \\
\hline $\mathrm{E}$ & 10 & 0.08 & 0.38 & 85 \\
\hline $\mathrm{F}$ & 7.5 & 0.03 & 0.12 & 44 \\
\hline $\mathrm{G}$ & 12.5 & 0.06 & 0.32 & 111 \\
\hline
\end{tabular}

[1] P. Helander, L.-G. Eriksson and F. Andersson, Plasma Phys. Control. Fusion 44, B247 (2002).

[2] M. N. Rosenbluth and S. V. Putvinski, Nucl. Fusion 37, 1355 (1997).

[3] L.-G. Eriksson, P. Helander, F. Andersson, D. Anderson, and M. Lisak, Phys. Rev. Lett. 92, $205004(2004)$.

[4] H. Smith, P. Helander, L.-G. Eriksson, D. Anderson, M. Lisak, and F. Andersson, Phys. Plasmas 13, 102502 (2006).

[5] Yu. A. Sokolov, JETP Lett. 29, 218 (1979).

[6] N. T. Besedin and I. M. Pankratov, Nucl. Fusion 26, 807 (1986).

[7] R. Jayakumar, H. H. Fleischmann, and S. J. Zweben, Phys. Lett. A172, 447 (1993).

[8] S. V. Putvinski, P. Barabaschi, N. Fujisawa et al., Plasma Phys. Control. Fusion 39, B157 (1997).

[9] A. Loarte, V. Riccardo, J. R. Martin-Solis, J. Paley, A. Huber, M. Lehnen and JET EFDA Contributors, Nucl. Fusion 51, 073004 (2011).

[10] P. Helander and D. J. Sigmar, Collisional transport in magnetized plasmas (Cambridge University Press, Cambridge, 2002).

[11] J. W. Connor, R. J. Hastie, Nucl. Fusion. 15, 415 (1975). 
[12] F. Andersson, P. Helander, and L.-G. Eriksson, Phys. Plasmas 8, 5221 (2001).

[13] J. Riemann, M. Borchardt, R. Schneider and A. Mutzke, J. Nucl. Mater. 337-339, 357-360 (2005).

[14] H. M. Smith and E. Verwichte, Phys. Plasmas 15, 072502 (2008). 